\title{
Identify More Non-Golay Complementary Sequences for OFDM with Low PMEPR Using Multi-dimensional Root Pairs
}

\author{
Yajun Wang, Cheng Zhao, Wen Chen and Jun Li, \\ Department of Electronic Engineering, Shanghai Jiaotong University, Minhang, Shanghai, China. \\ Email: $\{$ wangyj1859, solarismaya, wenchen, jleesr80, $@$ sjtu.edu.cn
}

\begin{abstract}
Recently, sub-root pairs and sequences are introduced to identify Davis-Jedwab (DJ) codes, non-Davis-Jedwab (non-DJ) Golay complementary sequences (GCS) and nonGolay complementary sequences (non-GCS) for OFDM with low PMEPR. In this paper, we extend sub-root pairs to superroot pairs. A discrete version of super-root pairs called multidimensional root pairs are used to build arbitrarily interleaving Boolean functions of long length. The newly identified arbitrarily interleaving Boolean functions can produce more non-DJ GCS and non-GCS with PMEPR at most pre-chosen positive number not always being a power of 2 . In this way, we propose an efficient method to identify more codes with low PMEPR for OFDM.
\end{abstract}

Index Term-Golay complementary sequences, OFDM, PMEPR, Root pair.

In multicarrier communications, the orthogonal frequency division multiplexing (OFDM) has been made use widely. However, a major drawback of OFDM signals is the high peak to mean envelope power ratio (PMEPR) of the uncoded OFDM signal. Several coding schemes to use the so-called Golay complementary sequences [1], [2], [3], [4] to encode the OFDM signals with PMEPR of at most 2 have been studied [5], [6], [7]. A main theoretical advance made by Davis and Jedweb [10] established a link between Golay's complementary sequences and certain second-order cosets of the generalized first-order Reed-Muller codes. A powerful code based on the union of these cosets can be used to perform error correction and ensures a PMEPR of at most 2. But the code rate rapidly decreases for larger block lengths. So Davis and Jedweb proposed including further cosets in order to increase the code rate at the cost of a slightly higher PMEPR [10]. Paterson's work [11] and more general study by Schmidt and Finger [16] provided a construction for further second-order cosets comprising of sequences lying in the complementary sets of size $2^{k+1}$. Thus the PMEPR of the code words in each coset of $R M_{q}(1, m)$ is at most $2^{k+1}$. But its upper bounds is not always tight. Another construction based on complementary sets was proposed by Parker and Tellambura [17], [18]. But in generally, the construction does not produce cosets of $R M_{q}(1, m)$.

Recently, sub-root pairs which is called seed extension in [15] are introduced to identify Davis-Jedwab (DJ)-codes, non-Davis-Jedwab (non-DJ) Golay complementary sequences (GCS) and non-Golay complementary sequences (non-GCS) for OFDM with low PMEPR [12]. However, the binary vari- ables of the sub-root pairs' Boolean function representations can not employ an arbitrarily interleaving pattern with those of DJ-codes. This is a drawback when come to the non-DJ Galay complementary sequences (GCS) which is discovered recently and explained in [8], [9]. In this paper, we generalize subroot pairs to super-root pairs which can be used to construct longer codes with low PMEPR for OFDM by any arbitrarily interleaving patten with DJ-codes. A discrete version of superroot pairs called multi-dimensional root pairs are used to build arbitrarily interleaving Boolean functions of long length, which can identify more non-DJ GCS and non-GCS with PMEPR at most pre-chosen positive number not always being power of 2 . In this way, we propose an efficient method to identify more codes with low PMEPR for OFDM.

\section{Notations AND PRELIMINARIES}

Before proceeding further, let us introduce the OFDM signals, the PMEPR and the related concepts at first. Throughout this paper $\xi=\exp (2 \pi j / M)$, where $M$ is a positive integer. Let an $M$-ary phase shift keying (MPSK) constellation be denoted by $\xi^{\mathbb{Z}_{M}}=\left\{\xi^{k}: k \in \mathbb{Z}_{M}\right\}$, where $\mathbb{Z}_{M}=$ $\{0, \cdots, M-1\}$.

\section{A. OFDM and Power Control}

Let $j$ be the imaginary unit, i.e., $j^{2}=-1$. For an MPSK modulation OFDM, let a codeword $\mathbf{c}=\left(c_{0}, \ldots, c_{n-1}\right)$ with $c_{\ell} \in \xi^{\mathbb{Z}_{M}}$, the frequency separation between any two adjacent subcarriers is $\Delta f=1 / T$. Then the $n$ subcarrier complex baseband OFDM signal can be represented as

$$
s_{\mathbf{c}}(t)=\sum_{\ell=0}^{n-1} c_{\ell} e^{j 2 \pi \ell \Delta f t},
$$

where $0 \leq t<T$. The instantaneous power of the complex envelope $s_{\mathbf{c}}(t)$ is defined by

$$
P_{\mathbf{c}}(t)=\left|s_{\mathbf{c}}(t)\right|^{2} .
$$

So the peak-to-mean power ratio (PMEPR) of the codeword $\mathbf{c}$ is defined by

$$
\operatorname{PMEPR}(\mathbf{c})=\frac{1}{n} \sup _{0 \leq t<T}\left|s_{\mathbf{c}}(t)\right|^{2} .
$$

Notice that the PMEPR can be as large as $n$, which occurs, for example, if $\mathbf{c}$ is the all-one word. However, it is desirable 
to use codewords with PMEPR that is substantially lower than $n$.

\section{B. Generalized Boolean Functions and Associated Sequences}

A generalized Boolean function $f$ is defined as a mapping $f: \mathbb{Z}_{2}^{m} \rightarrow \mathbb{Z}_{M}$. Such a function can be written uniquely in its algebraic normal form, i.e., $f$ is the sum of weighted monomials

$$
f\left(x_{0}, x_{1}, \ldots, x_{m-1}\right)=\sum_{i=0}^{2^{m}-1} c_{i} \prod_{\ell=0}^{m-1} x_{\ell}^{i_{\ell}},
$$

where the weights $c_{o}, \ldots, c_{2^{m}-1}$ are in $\mathbb{Z}_{M}$, and $\left(i_{0}, i_{1}, \ldots, i_{m-1}\right)$ is the binary representation of $i, 0 \leq i<2^{m}$, i.e., $i=\sum_{\ell=0}^{m-1} i_{\ell} 2^{\ell}$. The order of the $i$ th monomial is defined to be $\sum_{\ell=0}^{m-1} i_{\ell}$, and the order or algebraic degree of a generalized Boolean function, denoted by $\operatorname{deg}(f)$, is defined to be the highest order of the monomials with a nonzero coefficient in the algebraic normal form of $f$.

A generalized Boolean function may be equally represented by sequences of length $2^{m}$. Denote $f_{i}=f\left(i_{0}, i_{1}, \ldots, i_{m-1}\right)$, where $\left(i_{0}, i_{1}, \ldots, i_{m-1}\right)$ is the binary representation of $i$, $0 \leq i<2^{m}$. Define the $\mathbb{Z}_{M}$-valued sequence associated with $f$ as $\psi(f) \triangleq\left(f_{0}, f_{1}, \ldots, f_{2^{m}-1}\right)$, and the polyphase sequence associated with $f$ as $\varphi(f) \triangleq\left(\xi^{f_{0}}, \xi^{f_{1}}, \ldots, \xi^{f_{2} m}-1\right)$. Denote $\psi\left(f_{i}\right) \triangleq f_{i}$ and $\varphi\left(f_{i}\right) \triangleq \xi^{f_{i}}$.

\section{Aperiodic Correlations and Golay Complementary Se- quences}

Let $\mathbf{a}=\left(a_{0} a_{1} \ldots a_{n-1}\right)$ and $\mathbf{b}=\left(b_{0} b_{1} \ldots b_{n-1}\right)$ be two complex vectors of length $n$, where $a_{i}, b_{i} \in \mathbb{C}, 0 \leq i<n-1$. For an integer $\ell$, define the aperiodic correlation by

$$
C(\mathbf{a}, \mathbf{b})(\ell) \triangleq \begin{cases}\sum_{i=0}^{n-\ell-1} a_{i+\ell} b_{i}^{*}, & \text { if } 0 \leq \ell<n \\ \sum_{i=0}^{n+\ell-1} a_{i} b_{i-\ell}^{*}, & \text { if }-n<\ell \leq 0 \\ 0, & \text { otherwise, }\end{cases}
$$

and the auto correlation by $A(\mathbf{b})(\ell) \triangleq C(\mathbf{b}, \mathbf{b})(\ell)$.

From (1), (2) and (4), it is an easy exercise to show that

$$
\begin{aligned}
P_{\mathbf{c}}(t) & =\left|S_{\mathbf{c}}(t)\right|^{2} \\
& =\sum_{\ell=1-n}^{n-1} A(\mathbf{c})(\ell) e^{j 2 \pi \ell \Delta f t} \\
& =A(\mathbf{c})(0)+2 \cdot R e \sum_{\ell=1}^{n-1} A(\mathbf{c})(\ell) e^{j 2 \pi \ell \Delta f t} .
\end{aligned}
$$

A $\xi^{\mathbb{Z}_{M}}$-sequence $c$ of length $n$ is called a Golay complementary sequence (GCS) [10] if there is a $\xi^{\mathbb{Z}_{M}}$-sequence $d$ of length $n$ such that

$$
A(\mathbf{c})(\ell)+A(\mathbf{d})(\ell)=2 n \delta(\ell),
$$

where the Dirac function $\delta(\ell)$ is defined as $\delta(0)=1$ and $\delta(\ell)=0$ for $\ell \neq 0$. It is easy to see that $P_{c}(t)+P_{d}(t)=2 n$, and hence $\operatorname{PMEPR}(c) \leq 2$, if $c$ is a GCS. The pair $(c, d)$ is called a Golay complementary pair (GCP). A large class of
GCS called DJ-codes can be constructed by Boolean functions. Let a $\operatorname{GCP}(c, d)$ of the form in [10], i.e.,

$$
\left\{\begin{array}{l}
c=\frac{M}{2} \sum_{k=1}^{m-1} y_{\pi(k)} y_{\pi(k+1)}+\sum_{k=1}^{m} c_{k} y_{k}+c_{0}, \\
d=c+c_{0}^{\prime}+\frac{M}{2} y_{\pi(1)}
\end{array},\right.
$$

where $\pi$ is a permutation of $\{1,2, \ldots, m\}$ and $c_{0}^{\prime}, c_{0}, c_{1}, \ldots, c_{m} \in \mathbb{Z}_{M}$. Then $(c, d)$ is a DJ-GCP and either $c$ or $d$ is a DJ-codes.

\section{SUPER-Root PAIRs And MUlti-Dimensional RoOT PAIRS.}

Sub-root pairs [12] called seed extension in [15] are used to construct long codes with low PMEPR by using short DJcodes. However, the binary variables of the sub-root pairs' Boolean function representations can not employ an arbitrarily interleaving pattern. Here we extend sub-root pairs to superroot pairs which is a sub-class of Reed-Muller codes. A discrete version of super-root pairs called multi-dimensional root pairs are used to build arbitrarily interleaving Boolean functions of long length. Firstly, the definition of super-root pairs and multi-dimensional root pairs are given below.

Definition 1: Let $f\left(x_{1}, x_{2}, \ldots, x_{m}\right)$ and $g\left(x_{1}, x_{2}, \ldots, x_{m}\right)$ be two Boolean functions over $\mathbb{Z}_{2}^{m} \rightarrow \mathbb{Z}_{M}$. Denote $\mathcal{N}=2^{m}$. Let $C$ denote the unit circle. If for any complex vector $\mathbf{Z}=$ $\left(z_{0}, z_{1}, \ldots, z_{\mathcal{N}-1}\right) \in C^{\mathcal{N}}$, there is

$$
\left|\varphi(f) \mathbf{Z}^{T}\right|^{2}+\left|\varphi(g) \mathbf{Z}^{T}\right|^{2} \leq \gamma \mathcal{N}
$$

for a given positive number $\gamma$, then $[f, g, \gamma, m]$ is called a superroot pair. $f$ and $g$ are both called super-root sequences.

Since $\mathbf{Z}$ could be any complex vector in $C^{\mathcal{N}}$, it is difficult to build super-root pairs. Instead, a discrete version of superroot pairs called multi-dimensional root pairs, which have the same good properties will be defined. Our main identification theorems will be based on multi-dimensional root pairs. At first, some notations are given here. Denote the matrix representation of an 1 -dimensional $\mathcal{L}$-length IDFT with an over sampling factor $\lambda$ as

$$
\mathbf{Q}_{\mathcal{L}}^{1}(\lambda) \triangleq\left[e^{j \frac{2 \pi(i-1)(j-1)}{\lambda \mathcal{L}}}\right]_{1 \leq i \leq 2,1 \leq j \leq \lambda \mathcal{L}} .
$$

Let $\otimes$ denote the tensor product of matrices. Then the matrix representation of an $s$-dimensional $\mathcal{L}$-length IDFT with an over sampling factor $\lambda$ is defined as

$$
\mathbf{Q}_{\mathcal{L}}^{s}(\lambda) \triangleq \underbrace{\mathbf{Q}_{\mathcal{L}}^{1}(\lambda) \bigotimes \mathbf{Q}_{\mathcal{L}}^{1}(\lambda) \bigotimes \ldots \mathbf{Q}_{\mathcal{L}}^{1}(\lambda) \bigotimes \mathbf{Q}_{\mathcal{L}}^{1}(\lambda)}_{\text {The number of } \mathbf{Q}_{\mathcal{L}}^{1}(\lambda) \text { is } s .} .
$$

It is easy to see that any vector $\mathbf{Z} \in C^{\mathcal{L}}$ could be represented by the transpose of one of the column vector chosen from $\mathbf{Q}_{\mathcal{L}}^{\infty}(\infty)$, where

$$
\mathbf{Q}_{\mathcal{L}}^{\infty}(\infty) \triangleq \lim _{s \rightarrow \infty, \lambda \rightarrow \infty} \mathbf{Q}_{\mathcal{L}}^{s}(\lambda)
$$

Furthermore, when $s$ and $\lambda$ are large enough, $\mathbf{Q}_{\mathcal{L}}^{s}(\lambda)$ is a good discrete and finite approximation of the above $\mathbf{Z}$. Hence we have the following definitions: 
Definition 2: Let $f\left(x_{1}, x_{2}, \ldots, x_{m}\right)$ and $g\left(x_{1}, x_{2}, \ldots, x_{m}\right)$ be two Boolean functions over $\mathbb{Z}_{2}^{m} \rightarrow \mathbb{Z}_{M}$. Denote $\mathcal{N}=2^{m}$. If for a given positive number $\gamma$,

$$
\left\|\varphi(f) \mathbf{Q}_{\mathcal{L}}^{m}(\lambda)\right\|^{2}+\left\|\varphi(g) \mathbf{Q}_{\mathcal{L}}^{m}(\lambda)\right\|^{2} \leq \gamma \mathcal{N},
$$

then $[f, g, \gamma, m]$ is called an $m$-dimensional root pair with over sampling factor $\lambda . f$ and $g$ are both called $m$-dimensional root sequences. Here $\|\cdot\|$ denotes the maximum norm of a vector, which takes the maximum absolute value of all elements of the vector.

Remark 1: In Definition 2, the dimension of $s$ is set to be the number of the Boolean functions $f$ 's or $g$ 's variables, i.e., $m$. This is because in the following identification scheme, this dimension is enough for any arbitrarily interleaving Boolean function scheme, as the maximum partition possibility of the variables of $f$ or $g$ is $m$.

Remark 2: From numerical computation, the oversampling factor $\lambda=4$ is enough to estimate the PMEPR of the multidimensional root pairs. We will use $\lambda=4$ in the following.

By Remark 1 and 2, let $s=m$ and $\lambda=4$ in Definition 1 . Then the discrete $m$-dimensional root pairs will be a discrete version of super-root pair defined in Definition 1.

\section{ARbitrarily INTERLEAVING BoOleAn FunCtions WITH LOW PMEPR}

In this section, some basic construction theorems and corollaries are presented to construct Boolean functions of long length for OFDM modulation scheme from short multidimensional root pairs, by arbitrarily interleaving pattern, which have the same PMEPR as the multi-dimensional root pairs. Firstly, some notations and definitions are given.

Let $k$ be an integer such that $0<k<m$. Arbitrarily take $i_{0}, i_{1}, \ldots, i_{k-1}$ such that $0 \leq i_{0}<i_{1}<\ldots<i_{k-1}<m$. Suppose that $j_{0}, j_{1}, \ldots, j_{m-k-1}$ are the rest indices and $0 \leq$ $j_{0}<j_{1}<\ldots<j_{m-k-1}<m$. Let $\mathbf{X}=\left(x_{i_{0}}, \ldots, x_{i_{k-1}}\right)$ and $\mathbf{Y}=\left(y_{j_{0}}, \ldots, y_{j_{m-k-1}}\right)$ be two binary vectors of length $k$ and $m-k$, respectively. Denote $\mathbf{X} \uplus \mathbf{Y}$ as the binary vector of length $m$ decided by interleaving $\mathbf{X}$ and $\mathbf{Y}$ according to their indices $\left\{i_{\ell}\right\}$ and $\left\{j_{\ell}\right\}$ from small numbers to large numbers. Denote

$$
\mathbf{U}_{i} \triangleq\left[e^{j \frac{2 \pi(i-1)(j-1)}{\lambda \mathcal{L}}}\right]_{0 \leq j \leq \mathcal{L}},
$$

and $\mathbf{U}_{i}(j)$ as the $j$ th element of $\mathbf{U}_{i}$, where $0 \leq i \leq \lambda \mathcal{L}$. Also denote $\mathbf{P}_{\mathcal{L}}(\lambda)=\left[\mathbf{U}_{i}^{T}\right]_{0 \leq i \leq \lambda \mathcal{L}}$ as the $\mathcal{L}$-length IDFT transformation with oversampling factor $\lambda$. Similarly to the propositions in [12], we have the following propositions.

Proposition 1: Let $f\left(x_{1}, x_{2}, \ldots, x_{m}\right)$ be a Boolean function over $\mathbb{Z}_{2}^{m} \rightarrow \mathbb{Z}_{M}$. Denote $\widehat{f}=-f\left(\widehat{x_{1}}, \widehat{x_{2}}, \ldots, \widehat{x_{m}}\right)$, where $\left(\widehat{x_{1}}, \widehat{x_{2}}, \ldots, \widehat{x_{m}}\right) \triangleq\left(1-x_{1}, 1-x_{2}, \ldots, 1-x_{m}\right)$ in the sense of module 2 . Then
1) $\left\|\varphi(f) \mathbf{Q}_{2^{m}}^{m}(\lambda)\right\|_{2}=\left\|\varphi(\widehat{f}) \mathbf{Q}_{2^{m}}^{m}(\lambda)\right\|_{2}$
2) $\left\|\varphi(f) \mathbf{P}_{\mathcal{L}}(\lambda)\right\|_{2}=\left\|\varphi(\hat{f}) \mathbf{P}_{\mathcal{L}}(\lambda)\right\|_{2}$,
3) $\left|\varphi(f) \mathbf{Z}^{T}\right|=\left|\varphi(\widehat{f}) \mathbf{Z}^{T}\right|$.

Proposition 2: If two $\xi^{\mathbb{Z}_{M}}$-sequences $c$ and $d$ of length $n$ form a Golay complementary pair, then

1) $\left\|\varphi(c) \mathbf{Q}_{2^{m}}^{m}(\lambda)\right\|_{2}^{2}+\left\|\varphi(\widehat{d}) \mathbf{Q}_{2^{m}}^{m}(\lambda)\right\|_{2}^{2}=2 n$,

2) $\left\|\varphi(c) \mathbf{P}_{\mathcal{L}}(\lambda)\right\|_{2}^{2}+\left\|\varphi(\widehat{d}) \mathbf{P}_{\mathcal{L}}(\lambda)\right\|_{2}^{2}=2 n$,

3) $\left|\varphi(c) \mathbf{Z}^{T}\right|^{2}+\left|\varphi(\widehat{d}) \mathbf{Z}^{T}\right|^{2}=2 n$.

Theorem 1: Let $[a, b, \gamma, k$,$] be a k$-dimensional root pair with oversampling factor $\lambda$, and $c(\mathbf{Y})$ and $d(\mathbf{Y})$ be a GCP's Boolean function representations of length $m-k$. Define

$$
\begin{aligned}
& f(\mathbf{X} \uplus \mathbf{Y})=\left\{\begin{array}{l}
a(\mathbf{X})+c(\mathbf{Y}), \text { if } c(\mathbf{Y})=d(\mathbf{Y}) \\
b(\mathbf{X})+c(\mathbf{Y}), \text { if } c(\mathbf{Y}) \neq d(\mathbf{Y})
\end{array}\right. \\
& g(\mathbf{X} \uplus \mathbf{Y})=\left\{\begin{array}{l}
a(\mathbf{X})+\widehat{d}(\mathbf{Y}), \text { if } c(\mathbf{Y}) \neq d(\mathbf{Y}) \\
b(\mathbf{X})+\widehat{d}(\mathbf{Y}), \text { if } c(\mathbf{Y})=d(\mathbf{Y})
\end{array}\right.
\end{aligned}
$$

Then $f(\mathbf{X} \uplus \mathbf{Y})$ and $g(\mathbf{X} \uplus \mathbf{Y})$ are two Boolean function representations of length $m$ with PMEPR $\leq \gamma$ for OFDM modulation with oversampling factor $\lambda$.

Proof: omitted due to limited space.

Remark 3: Theorem 1 indicates that in order to construct long Boolean function with PMEPR $\leq \gamma$ a pre-chosen positive number not always being power of 2 , we have to find two short $k$-dimensional root pairs. This can be attained by an exhaustive search if $k$ is small enough. An example will be given in Section V.

In order to derive an explicit Boolean function for $f$ and $g$, DJ-codes are used instead of $c$ and $d$ in Theorem 1. Similar approach was used in [8] and [9]. Then we have the following two corollaries:

Construction 1: Theorem 1 holds if

$$
\left\{\begin{array}{l}
f(\mathbf{X} \uplus \mathbf{Y})=a(\mathbf{X}) \widehat{y_{\pi(1)}}+b(\mathbf{X}) y_{\pi(1)}+c(\mathbf{Y}) \\
g(\mathbf{X} \uplus \mathbf{Y})=f(\mathbf{X} \uplus \mathbf{Y})+\frac{M}{2} y_{\pi(m-k)}+e
\end{array} .\right.
$$

Similarly to [12], the following construction produces more sequences.

Construction 2: Theorem 1 holds if

$$
\left\{\begin{aligned}
f(\mathbf{X} \uplus \mathbf{Y})= & a(\mathbf{X}) \widehat{y_{\pi(\ell)}} \widehat{y_{\pi(\ell-1)}}+b(\mathbf{X}) \widehat{y_{\pi(\ell)}} y_{\pi(\ell-1)} \\
& +\widehat{b}(\mathbf{X}) y_{\pi(\ell)} \widehat{y_{\pi(\ell-1)}}+\widehat{a}(\mathbf{X}) y_{\pi(\ell)} y_{\pi(\ell-1)} . \\
& +c(\mathbf{Y}) \\
g(\mathbf{X} \uplus \mathbf{Y})= & f(\mathbf{X} \uplus \mathbf{Y})+\frac{M}{2} y_{\pi(m-k)}+e
\end{aligned}\right.
$$

To discern the multi-dimensional sequences identified by the two Constructions. Let $\left(a, a^{\prime}\right)$ be an $m$-dimensional root pair over $\mathbb{Z}_{2}^{m} \rightarrow \mathbb{Z}_{M}$. Denote

$$
E(a) \triangleq\left\{a+e, \widehat{a}+e: e \in \mathbb{Z}_{M}\right\} .
$$

Let $c \in E(a)$ and $d \in E\left(a^{\prime}\right)$ form an $m$-dimensional root pair. If $E(a) \neq E\left(a^{\prime}\right)$, then we call $c$ and $d$ form $m$ dimensional root pair by a cross-over of their autocorrelation functions [8], [9]. In this case, $(c, d)$ is also called a nonstandard $m$-dimensional root pair, and $c$ and $d$ are both called non-standard $m$-dimensional root sequences; Otherwise, $(c, d)$ is called a standard $m$-dimensional root pair, and $c$ and $d$ are both called standard $m$-dimensional root sequences. 


\section{LOW PMEPR CODES FOR OFDM}

In this section, we will identify long codes with low PMEPR for OFDM using the above Construction 1 and 2. It is obvious that the degrees of the identified $f$ and $g$ are respectively (by Construction 1)

$$
\max \{\operatorname{deg}(f)\}=\max \{\operatorname{deg}(g)\}=\max \{\operatorname{deg}(a)\}+1 ;
$$

and (by Construction 2)

$$
\max \{\operatorname{deg}(f)\}=\max \{\operatorname{deg}(g)\}=\max \{\operatorname{deg}(a)\}+2 .
$$

Firstly, we get all of the 2-dimensional and 3-dimensional codes represented by the Boolean functions $f: \mathbb{Z}_{2}^{2} \rightarrow \mathbb{Z}_{M}$ and $f: \mathbb{Z}_{2}^{3} \rightarrow \mathbb{Z}_{M}$ with PMEPR less than a pre-chosen $\gamma$ by exhaustive computer search. Then we use them to identify more codes with PMEPR controlled by $\gamma$ for OFDM which lie in the third, fourth and fifth order cosets of the first-order Reed-Muller codes. Let

$$
f\left(x_{1}, x_{2}\right)=C_{0}+C_{1} x_{1}+C_{2} x_{2}+C_{3} x_{1} x_{2},
$$

where $C_{i} \in \mathbb{Z}_{M}, x_{i} \in \mathbb{Z}_{2}$. Then we obtain a sequence

$$
\left(C_{0}, C_{0}+C_{1}, C_{0}+C_{2}, C_{0}+C_{1}+C_{2}+C_{3}\right) .
$$

It is straightforward to see that the vector minus $C_{0}$ from each element can represent these different sequences with the same PMEPR [8]. So it's enough to use only $\left[C_{1}, C_{2}, C_{3}\right]$ to represent these sequences, while $C_{0}$ is any element in $\mathbb{Z}_{M}$. So is $\left[C_{1}, C_{2}, C_{3}, C_{4}, C_{5}, C_{6}, C_{7}\right]$ when considering $f: \mathbb{Z}_{2}^{3} \rightarrow$ $\mathbb{Z}_{M}$.

In order to present some construction examples, the initial $[a, b, 5,2] 2$-dimensional root pairs with 28 pairs representatives are listed in Table I. Choose a root pair $((0,0,1),(1,1,0))$ from Table I, i.e.,

$$
\left\{\begin{array}{l}
a\left(x_{1}, x_{2}\right)=x_{1} x_{2} \\
b\left(x_{1}, x_{2}\right)=x_{1}+x_{2} .
\end{array}\right.
$$

Then

$$
\left\{\begin{array}{l}
\hat{a}\left(x_{1}, x_{2}\right)=1+x_{1}+x_{2}+x_{1} x_{2}, \\
\hat{b}\left(x_{1}, x_{2}\right)=x_{1}+x_{2} .
\end{array}\right.
$$

We have

$$
\operatorname{Max}\left\{\left\|\varphi(a) \mathbf{Q}_{4}^{2}(4)\right\|_{2}^{2}+\left\|\varphi(b) \mathbf{Q}_{4}^{2}(4)\right\|_{2}^{2}\right\} \leq 4 \cdot 2^{2} .
$$

In order to construct sequences $f$ and $g$ of length $2^{4}$, let $c$ be

\begin{tabular}{|c|c|c|c|c|c|c|}
\hline \multicolumn{3}{|c|}{$a$} & \multicolumn{3}{|c|}{$b$} & \multirow{2}{*}{$\begin{array}{l}\frac{1}{4} \operatorname{Max}\left\{\left\|\varphi(a) \mathbf{Q}_{4}^{2}(4)\right\|_{2}^{2}\right. \\
\left.+\left\|\varphi(b) \mathbf{Q}_{4}^{2}(4)\right\|_{2}^{2}\right\}\end{array}$} \\
\hline$\overline{a_{1}}$ & $a_{2}$ & $a_{3}$ & $b_{1}$ & $b_{2}$ & $b_{3}$ & \\
\hline 0 & 0 & 0 & 0 & 0 & 1 & \\
\hline 0 & 0 & 0 & 0 & 1 & 0 & 4 \\
\hline 0 & 0 & 0 & 0 & 1 & 1 & 5 \\
\hline 0 & 0 & 0 & 1 & 0 & 0 & 4 \\
\hline 0 & 0 & 0 & 1 & 0 & 1 & 5 \\
\hline 0 & 0 & 0 & 1 & 1 & 0 & 4 \\
\hline 0 & 0 & 0 & 1 & 1 & 1 & 5 \\
\hline 0 & 0 & 1 & 0 & 1 & 0 & 5 \\
\hline 0 & 0 & 1 & 0 & 1 & 1 & 2 \\
\hline 0 & 0 & 1 & 1 & 0 & 0 & 5 \\
\hline 0 & 0 & 1 & 1 & 0 & 1 & 2 \\
\hline 0 & 0 & 1 & 1 & 1 & 0 & 5 \\
\hline 0 & 0 & 1 & 1 & 1 & 1 & 4 \\
\hline 0 & 1 & 0 & 0 & 1 & 1 & 5 \\
\hline 0 & 1 & 0 & 1 & 0 & 0 & 4 \\
\hline 0 & 1 & 0 & 1 & 0 & 1 & 5 \\
\hline 0 & 1 & 0 & 1 & 1 & 0 & 4 \\
\hline 0 & 1 & 0 & 1 & 1 & 1 & 5 \\
\hline 0 & 1 & 1 & 1 & 0 & 0 & 5 \\
\hline 0 & 1 & 1 & 1 & 0 & 1 & 4 \\
\hline 0 & 1 & 1 & 1 & 1 & 0 & 5 \\
\hline 0 & 1 & 1 & 1 & 1 & 1 & 2 \\
\hline 1 & 0 & 0 & 1 & 0 & 1 & 5 \\
\hline 1 & 0 & 0 & 1 & 1 & 0 & 4 \\
\hline 1 & 0 & 0 & 1 & 1 & 1 & 5 \\
\hline 1 & 0 & 1 & 1 & 1 & 0 & 5 \\
\hline 1 & 0 & 1 & 1 & 1 & 1 & 2 \\
\hline 1 & 1 & 0 & 1 & 1 & 1 & 5 \\
\hline
\end{tabular}
a DJ-code over $\mathbb{Z}_{2}^{2} \longrightarrow \mathbb{Z}_{2}$, i.e.,

$$
c\left(y_{1}, y_{2}\right)=c_{0}+c_{1} y_{1}+c_{2} y_{2}+y_{\pi(1)} y_{\pi(2)} .
$$

Applying Construction 1 to $\langle a, b\rangle$, we have

$$
\begin{array}{r}
f=x_{1} x_{2}+x_{1} y_{\pi(1)}+x_{2} y_{\pi(1)}+x_{1} x_{2} y_{\pi(1)}+c_{0}+c_{1} y_{1} \\
+c_{2} y_{2}+y_{\pi(1)} y_{\pi(2)} .
\end{array}
$$

TABLE I

THE INITIAL $[a, b, 5,2]$ ROOT WITH 28 PAIRS REPRESENTATIVES.

Applying Construction 2 to $\langle a, b\rangle$, we have

$$
\begin{aligned}
f= & x_{1} x_{2}+x_{1} y_{\pi(\ell-1)}+x_{2} y_{\pi(\ell-1)}+x_{1} x_{2} y_{\pi(\ell-1)} \\
& +x_{1} y_{\pi(\ell)}+x_{2} y_{\pi(\ell)}+x_{1} x_{2} y_{\pi(\ell)}+y_{\pi(\ell-1)} y_{\pi(\ell)} \\
& +x_{1} y_{\pi(\ell-1)} y_{\pi(\ell)}+x_{2} y_{\pi(\ell-1)} y_{\pi(\ell)}+c_{0}+c_{1} y_{1} \\
& +c_{2} y_{2}+y_{\pi(1)} y_{\pi(2)} .
\end{aligned}
$$

By numerical computation, such a 2-dimensional root pair's four ordered pairwise pattern, i.e., $\langle a, b\rangle,\langle b, a\rangle,\langle\hat{a}, \hat{b}\rangle$ and $\langle\hat{b}, \hat{a}\rangle$ can together generate 384 and 192 non-DJ codes $f$ or $g$ of length 16 by Construction 1 and Construction 2, respectively. By one-dimensional method [12], we can only produce 192 and 96 non-DJ codes respectively. It follows that Multi-dimensional pairs can produce more codes for OFDM compared with sub-root pairs, because it allows arbitrarily interleaving Boolean functions schemes.

In order to explain the performance of the arbitrarily interleaving Boolean functions for OFDM, we list some of the numbers of the identified higher order non-DJ codes by 2dimensional and 3-dimensional root pairs and the improved OFDM code rates in Table II, for 8,16 , and 32 sub-carriers respectively, which have some performance gain compared to the previous works [12].

It is noticeable that, in some cases, the new proposed multi-dimensional scheme will identify more non-DJ codes for OFDM than the previously proposed sub-root pairs in [12] with the same PMEPR. An interesting example is already 
TABLE II

ENUMERATE THE IDENTIFIED NON-DJ CODES BY 2-DIMENSIONAL AND 3-DIMENSIONAL ROOT PAIRS AND THE IMPROVED OFDM CODE RATES.

\begin{tabular}{|c|c|c|c|c|}
\hline $\begin{array}{c}\text { OFDM } \\
\text { Carriers }\end{array}$ & DJ-codes & $\begin{array}{c}\text { Identified } \\
\text { non-DJ codes }\end{array}$ & $\begin{array}{c}\text { Old } \\
\text { code rate }\end{array}$ & $\begin{array}{c}\text { New } \\
\text { code rate }\end{array}$ \\
\hline \multicolumn{5}{|c|}{$\begin{array}{c}\text { The identified codes lie in cosets of } R M_{M}(1, m) \text { in } R M_{M}(3, m) \\
\text { using Construction } 1 \text { with PMEPR } \leq 4 .\end{array}$} \\
\hline 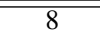 & 48 & 24 & $\overline{0.6981}$ & 0.7712 \\
\hline 16 & 384 & 362 & 0.5366 & 0.5964 \\
\hline 32 & 3840 & 6240 & 0.3721 & 0.4156 \\
\hline \multicolumn{5}{|c|}{$\begin{array}{c}\text { The identified codes lie in cosets of } R M_{M}(1, m) \text { in } R M_{M}(4, m) \\
\text { using Construction } 1 \text { with PMEPR } \leq 4\end{array}$} \\
\hline 8 & 48 & 26 & 0.6981 & 0.7762 \\
\hline 16 & 384 & 5124 & 0.5366 & 0.7767 \\
\hline 32 & 3840 & 68052 & 0.3721 & 0.5042 \\
\hline \multicolumn{5}{|c|}{$\begin{array}{c}\text { The identified codes lie in cosets of } R M_{M}(1, m) \text { in } R M_{M}(3, m \\
\text { using Construction } 1 \text { with PMEPR } \leq 5 .\end{array}$} \\
\hline$\overline{88}$ & $\overline{488}$ & 192 & $\overline{0.6981}$ & 0.9884 \\
\hline 16 & 384 & 4010 & 0.5366 & 0.7563 \\
\hline 32 & 3840 & 42720 & 0.3721 & 0.4848 \\
\hline \multicolumn{5}{|c|}{$\begin{array}{c}\text { The identified codes lie in cosets of } R M_{M}(1, m) \text { in } R M_{M}(4, m) \\
\text { using Construction } 1 \text { with PMEPR } \leq 5 .\end{array}$} \\
\hline 8 & $\overline{48}$ & $\overline{192}$ & 0.6981 & 0.9884 \\
\hline 16 & 384 & 15408 & 0.5366 & 0.8717 \\
\hline 32 & 3840 & 583760 & 0.3721 & 0.5989 \\
\hline
\end{tabular}

shown above. We will give more detailed explanations. From Table ??, when we let the PMEPR be 5 , the number of sub-root pairs of length 2 is the same as the number of 2 dimensional root pairs, i.e., 28. As the 2-dimensional root pairs introduce arbitrarily interleaving Boolean function scheme, we can produce more codes with the same PMEPR for OFDM. By numerical results, when these 28 pairs are considered as sub-root pairs, the numbers of the identified generalized first order Reed-Muller codes lying in the third order non-DJ ReedMuller codes are 192, 2198, and 18240 for 8,16 , and 32 subcarriers respectively with PMEPR $\leq 5$. On the other hand, when they are considered as the 2-dimensional root pairs, the numbers of the identified generalized first order ReedMuller codes lying in the third order non-DJ Reed-Muller codes are 192, 4010, and 42720 for 8, 16, and 32 sub-carriers respectively with the same PMEPR.

\section{CONCLUSION}

In this paper, we extend sub-root pairs to super-root pairs. A discrete version of super-root pairs called multi-dimensional root pairs are used to build arbitrarily interleaving Boolean functions with long length. The new identified arbitrarily interleaving Boolean functions can produce more non-DJ GCS and non-GCS with PMEPR at most a pre-chosen positive number not always being power of 2 . In this way, we propose an efficient method to identify more codes with low PMEPR for OFDM.

\section{ACKNOWLEDGMENT}

This work is supported by NSF China \#60672067, by NSF Shanghai \#062R14041, by Shanghai-Canada NRC \#06SN07112, by Cultivation Fund of the Key Scientific and Technical Innovation Project, Ministry of Education of China
\#706022, and by Program for New Century Excellent Talents in University (NCET).

\section{REFERENCES}

[1] M. J. E. Golay, "Complementary series."IRE Trans.Inf.theory, vol. IT-7, no. 2, pp. 82-87, Apr. 1961.

[2] W. Chen, and C. Tellambura, A Novel Complementary Sequence and PMEPR Reduction in OFDM, Canadian Journal of Electrical and Computer Engineering, vol. 33, no. 1, pp. 45-51, 2008.

[3] W. Chen, and C. Tellambura A good trade-off performance between the code rate and the PMEPR for OFDM signals using the generalized RudinShapiro polynomials, IEEE International Conference on Communications (ICC), pp. 2600-2604, 2005. (Seoul, Korea).

[4] W. Chen, and C. Tellambura, Identifying a class of multiple shift complementary sequences in the second order cosets of the first order Reed-Muller codes, IEEE International Conference on Communications (ICC), pp. 618-621, 2005. (Seoul, Korea)

[5] R. D. J. van Nee, "OFDM codes for peak-to-average power reduction and error correction."Proc. IEEE Global Telecommunications Conference (GLOBECOM), London, U.K., Nov. 1996, pp. 740-744.

[6] T. A. Wilkinson, and A. E. Jones, "Minimization of the peak to mean envelop power ratio of multicarrier transmission schemes by block coding," Proc. IEEE Vehicular Technology Conference (VTC), Chicago, IL, Jul. 1995, pp. 825-829.

[7] H. Ochiai and H. Imai, "Block coding scheme based on complementary sequences for multicarrier signals," IEICE Trans. Fundamentals, vol. E80A, pp. 2136-2146, 1997.

[8] F. Fiedler and J. Jedwab, "How do more Golay sequences arise?" IEEE Trans. Information Theory. vol. 52, pp. 4261-4266, 2006.

[9] F. Fiedler, J. Jedwab, and M. G. Parker, "A Framework for the Construction of Golay Sequences," IEEE Trans. Information Theory. vol. 54, no. 7 , pp. 3124-3129, July 2008.

[10] J. A. Davis, and J. Jedwab, "Peak to mean power control in OFDM, Golay complementary sequences and Reed-Miller codes," IEEE Trans. Inform. Theory, vol. 45, no. 7, pp. 2397-2417, Nov., 1999.

[11] K. G. Paterson, "Generalized Reed-Muller code and power control in OFDM modulation," IEEE Trans. Information Theory, vol. 46, no. 1, pp. 104-120, Jan., 2001.

[12] C. Zhao, W. Chen, and H. Shen, "A New Scheme To Identify Good Codes For OFDM with Low PMEPR," in Proc. of IEEE International Conference on Acoustics, Speech and Signal Processing (ICASSP). pp. 365-368, April 15-20, 2007, (Honolulu, Hawaii, USA).

[13] K. U. Schmidt, "On cosets of the generalized first-order Reed-Muller code with low PMEPR," IEEE Trans.Information Theory, vol. 52, no. 7, pp. 3220-3232, July, 2006.

[14] F. Fiedler, J. Jedwab, and M. G. Parker, "A multi-dimensional approach to the construction and enumeration of Golay complementary sequences," Journal of Combinatorial Theory (SeriesA), vol. 115, no. 5, pp. 753-776, 2008.

[15] M. G. Parker, and C. Tellambura, "Generalised Rudin-Shapiro constructions." In International Workshop on Coding and Cryptography (WCC), Paiis, France, 2001, [Online]. Available: http://www.ii.uib.no/matthew/ConstaBent2.pdf.

[16] K. U. Schmidt, and Adolf Finger "Construction of Complementary Sequences for Power-Controlled OFDM Transmission," In Proc. Workshop on Coding and Cryptography (WCC), 2005. (Lecture Notes in Computer Science), Berlin, Germany: Springer-Verlag, 2006.

[17] M. G. Parker, and C. Tellambura, "A Construction for Binary Sequences Sets with Low Peak-to-Average Power Ratio," Int. symp. Information Theory, Lausanne, Switzerland, pp. 239, June 30-July 5, 2002.

[18] M. G. Parker and C. Tellambura, "A Construction for Binary Sequences Sets with Low Peak-to-Average Power Ratio," Reports in informatics, University of Bergen, Report No242, ISSN 0333-3590, February 2003. 